\title{
Vacunación de gripe en niños con diagnóstico de diabetes mellitus tipo 1 en un área suburbana de Madrid
}

\author{
MJ. Rivero Martín, MJ. Alcázar Villar, D. Montes Bentura, \\ C. Navarro Moreno, M. Sanz Fernández
}

Servicio de Pediatría. Hospital Universitario de Fuenlabrada. Fuenlabrada, Madrid. España.

\section{Resumen}

Objetivo: determinar la tasa de vacunación frente a la gripe en el grupo de pacientes con diabetes mellitus (DM) tipo 1 en seguimiento en nuestro centro.

Material y métodos: en los pacientes con diagnóstico de DM tipo 1 se realiza un estudio transversal descriptivo analizando la tasa de vacunación frente a la gripe.

Resultados: se incluyen 37 pacientes (varones/mujeres: 1,6/1). La edad media es 9,7 años y el tiempo de evolución de la enfermedad tres años. El 51,3\% ha sido vacunado contra la gripe en 2010, frente al $80 \%$ en 2009.

Los niños con un tiempo de evolución entre tres y seis años son los menos vacunados (22\% de los vacunados), frente al resto (56\%).

Conclusiones: la cobertura vacunal frente al virus de la gripe es escasa en nuestro medio, aunque superior a la referida para el territorio español (36,7\%). Es necesaria la recomendación de la vacunación antigripal a pacientes diabéticos.

Palabras clave: Gripe. Diabetes mellitus. Vacunación. Cobertura vacunal.

Influenza vaccination in children with type I mellitus diabetes in a suburban area in Madrid

Abstract

Objective: To determine the rate of flu vaccination in the group of patients followed in our hospital because of type 1 diabetes.

Methods: A descriptive study is conducted in patients with type 1 diabetes, analyzing the rate of vaccination against influenza.

Results: 37 patients were included (male/female: 1.6/1). The mean age was 9.7 years and the mean duration of disease 3 years. $51.3 \%$ patients have been vaccinated against influenza in 2010 versus $64.86 \%$ in 2009. Children with a disease evolution between 3 and 6 years are the least vaccinated (22\% vaccinated), compared to the rest (56\%).

M. ${ }^{a}$ José Rivero Martín, mrivero.hflr@salud.madrid.org

Los autores declaran no presentar conflictos de intereses en relación con la preparación y publicación de este artículo. 
Conclusions: Vaccination coverage against influenza virus is low in our area, although higher than the referred in Spanish population (36.7\%). Recommendation on influenza vaccination is required in diabetic patients.

Key words: Influenza. Diabetes. Vaccination. Vaccination coverage.

\section{Introducción}

Los pacientes con diabetes mellitus (DM) son uno de los llamados "grupos de riesgo" para el padecimiento de la infección por el virus de la gripe. En España, las recomendaciones para la vacunación antigripal incluyen a niños de seis meses de edad o mayores con enfermedades crónicas pulmonares o cardiovasculares; a niños de cualquier edad a partir de seis meses, residentes en instituciones cerradas; a niños de seis meses de edad o mayores que precisaron tratamiento médico regular u hospitalización durante el año anterior a causa de enfermedades metabólicas crónicas (incluida DM), insuficiencia renal, hemoglobinopatías o inmunosupresión; y a niños y adolescentes con tratamientos prolongados con ácido acetilsalicílico'.

Se ha comprobado que las infecciones por el virus de la gripe tienen mayor morbilidad, tasa de hospitalización y mortalidad en niños con enfermedades crónicas que en niños sanos ${ }^{2}$. Durante la epidemia de gripe H1N1, el riesgo de hospitalización y de ingreso en la Unidad de Cuidados Intensivos se triplicó y cuadruplicó, respectivamente, en la población diabética ${ }^{3}$. A pesar de ello, muchos de estos grupos de riesgo no tienen una buena cobertura vacunal.

El objetivo principal de este trabajo ha sido determinar la tasa de vacunación contra la gripe en el grupo de pacientes con DM tipo 1 en seguimiento en el Servicio de Pediatría, área de Endocrinología, del Hospital Universitario de Fuenlabrada. Como objetivo secundario, se buscó determinar si el tiempo de evolución de la enfermedad, la edad y el origen modifican esta tasa de vacunación.

\section{Material y métodos}

Se ha llevado a cabo un estudio transversal descriptivo.

La campaña vacunal de gripe en la Comunidad de Madrid en el año 2010 se inició el 1 de octubre y finalizó el 30 de noviembre, aunque los pacientes que lo requirieran podrían vacunarse posteriormente a esta fecha. La tasa de vacunación frente a gripe $A$ se estudió a fecha 31 de diciembre de 2010. El acceso al estado vacunal se llevó a cabo por 
consulta de la historia clínica informatizada.

El grupo de estudio fue el de los pacientes con diagnóstico de DM tipo 1 y que han tenido seguimiento en el Servicio de Pediatría del Hospital de Fuenlabrada durante el año 2010.

\section{Resultados}

De los 42 pacientes con DM tipo 1 en seguimiento, tres han pasado a la consulta de adultos y dos han dejado de asistir a nuestro centro. En total, han sido atendidos en la consulta de diabetes infantojuvenil 37 pacientes en el año 2010; siete pacientes han sido diagnosticados entre el 1 de enero y el 31 de diciembre de 2010 (18,9\%).

De los pacientes, 23 eran varones (62\%) y 14 mujeres (38\%). La edad media de los pacientes es de 9,7 años (desviación estándar [DE]: 4,04), y el tiempo medio de evolución de la enfermedad, tres años (DE: 2,4). El 62,2\% (23 pacientes) de los niños tenía diagnóstico de DM tipo 1 desde hacía menos de tres años; el $18,9 \%$, desde hacía entre tres y seis años (siete pacientes), y otro $18,9 \%$, desde hacía más de seis años.

De los 37 pacientes, solo 19 (51,3\%) han sido vacunados contra la gripe en el año 2010. En el año 2009, de los 30 pacientes con diabetes mellitus tipo 1, 24 $(80 \%)$ fueron vacunados frente a gripe $(p<0,05)$ (tabla 1).

No hay diferencias en cuanto a la edad entre los vacunados y los no vacunados.

Los niños con un tiempo de evolución entre los tres y los seis años son los menos vacunados $(28 \%)$, frente a los de menos de tres años de evolución (56\%) y los de más de seis años (57\%).

El $75 \%$ de los pacientes son de familias originarias de España, y el 25\%, de otros países. Dentro de la población inmigrante, el $22,1 \%$ se ha vacunado de gripe, frente al $62,9 \%$ de los españoles, no encontrando diferencias estadísticamente significativas $(p=0,08)$.

\section{Discusión}

La mitad de los niños diabéticos de nuestro medio han sido vacunados fren-

Tabla 1. Vacunación frente a la gripe en niños diabéticos (diabetes mellitus tipo 1) en 2009 y 2010

\begin{tabular}{l|c|c|c}
\hline Vacunación frente a la gripe & \multicolumn{2}{|c|}{ Año (número de pacientes) } & \multirow{2}{*}{$\begin{array}{c}\text { Significación } \\
\text { estadística }\end{array}$} \\
\cline { 2 - 3 } en niños con DM tipo 1 & $\mathbf{2 0 1 0 ( 3 7 )}$ & $\mathbf{2 0 0 9 ( 3 0 )}$ & $\mathrm{P}<0,5$ \\
\hline Sí (\%) & $19(51,35 \%)$ & $24(80 \%)$ & $\mathrm{P}<0,5$ \\
\hline No $(\%)$ & $18(48,65 \%)$ & $6(20 \%)$ & \\
\hline
\end{tabular}

DM: diabetes mellitus. 
te a la gripe en 2010, una de las causas de esta baja tasa de vacunación puede ser la falta de conciencia de los riesgos relacionados con las infecciones por gripe. Por otro lado, debemos tener en cuenta que la administración de las vacunas se realiza habitualmente en los centros de salud de Atención Primaria y el seguimiento de la enfermedad crónica en Atención Especializada, de esta mane$\mathrm{ra}$, la falta de comunicación y de continuidad asistencial puede derivar en que no se realicen las actividades preventivas pertinentes ${ }^{4}$. Por otro lado, en muchas ocasiones los servicios hospitalarios muestran carencias en los conocimientos sobre vacunaciones ${ }^{5,6}$. En relación con las recomendaciones de salud, estas deben ser uniformes entre niveles asistenciales, ya que existe un riesgo importante de que mensajes contradictorios disminuyan la confianza de los pacientes.

A pesar de que solo el 51,3\% de nuestros pacientes se haya vacunado en 2010 , estos resultados de cobertura vacunal son superiores a los alcanzados en pacientes diabéticos de seis meses a 16 años en el conjunto del territorio español $(36,7 \%)^{7,8}$, aunque similares a datos de niños diabéticos italianos $(61 \%)^{9}$.

Hay una diferencia estadísticamente significativa entre las vacunaciones del año 2009 y las del 2010 (51,3\% vacuna- dos frente a $80 \%$ ). Es llamativo el comportamiento desigual en años consecutivos para la misma población de niños diabéticos y los mismos médicos encargados de su seguimiento, tanto a nivel hospitalario como de Atención Primaria; es probable que este hecho tenga relación con la campaña de vacunación y la alerta social que generó la gripe por el virus H1N1 en el otoño-invierno de 2009, de tal manera que tanto clínicos como población general estuvieron más sensibilizados frente a la vacunación.

La diferencia de la tasa de vacunación entre población inmigrante y no inmigrante no es significativa $(p=0,08)$, siendo concordante con los resultados de estudios españoles de vacunación antigripal en pacientes inmigrantes frente a pacientes locales, donde tampoco se obtuvieron diferencias significativas ${ }^{10}$. Sin embargo, debemos tener en cuenta que en nuestro trabajo el tamaño muestral (37 pacientes) es pequeño y no suficiente para establecer esas diferencias.

\section{Conclusiones}

La cobertura de vacunación frente al virus de la gripe es escasa en nuestro medio, aunque superior a la referida para el territorio español.

Es necesario hacer hincapié en la vacunación antigripal dentro de las recomen- 
daciones generales a pacientes diabéticos, así como comprobar su administración en las visitas programadas.

La coordinación entre Atención Primaria y Especializada es clave en el seguimiento de pacientes crónicos.
Es preciso establecer si en niños diabéticos las ventajas de la vacunación antigripal son las mismas que en la población adulta, donde la infección por el virus de la gripe aumenta claramente la morbilidad y la mortalidad.

\section{Bibliografía}

1. Marés Bermúdez J, Van Esso Arbolave $D$, Moreno Pérez D, Merino Moína M, Álvarez García FJ, Cilleruelo Ortega MJ y cols. Calendario de vacunaciones de la Asociación Española de Pediatría: recomendaciones 2011. An Pediatr (Barc). 2011;74:132e1-133e19.

2. Bhat N, Wright JG, Broder KR, Murray EL, Greenberg ME, Glover MJ et al. Influenza-Associated Deaths among Children in the United States, 2003-2004. N Engl J Med. 2005;353:2559-67.

3. Allard R, Leclerc $P$, Tremblay C, Tannenbaum TN. Diabetes and the severity of pandemic influenza $A$ (H1N1) infection. Diabetes Care. 2010;33(7):1491-3.

4. Giannattasio $A$, Lo Vecchio $A$, Franzese $A$, Prisco $F$, Femiano $P$, Guarino A. Redundancy of roles by physicians in charge of paediatric diabetes is a barrier to flu immunisation. Arch Dis Child. 2010;95(5):399-400.

5. Esposito $S$, Tremolati E, Bellasio M, Chiarelli G, Marchisio P, Tiso B, and the VIP Study Group. Attitudes and knowledge regarding influenza vaccination among hospital health workers caring for women and children. Vaccine. 2007;25:5283-9.
6. Davies P, Nwokoro Ch, Leight M. Vaccinations against influenza and pneumococcus in children with diabetes: telephone questionnaire survey. BMJ. 2004;328:203.

7. López de Andrés A, Hernández Barrera $V$, Carrasco Garrido P, Gil de Miguel A. Influenza vaccination coverage among Spanish children, 2006. Public Health. 2009;123:465-9.

8. Vázquez-Fernández del Pozo S, Hernández-Barrera V, Carrasco Garrido PE, ÁlvarezMartín E, López de Andrés A, Gil de Miguel A et al. Influenza vaccination coverage and related factors among Spanish children. J Infect. 2007; 54:483-9.

9. Giannattasio $A$, Squeglia $V$, Lo Vechio $A$, Russo MA, Barbarino $A$, Carlomagno $R$ et al. Pneumococcal and influenza vaccination rates and their determinants in children with cronic medical conditions. Italian J Pediatr. 2010;36:28.

10. Jiménez-García R, Hernández-Barrera $V$, Carrasco-Garrido $P$, de Andrés AL, Esteban y Peña $M M$, de Miguel AG. Coverage and predictors of Influenza vaccination among adults living in a large metropolitan area in Spain: a comparison between the inmigrant and indigenous populations. Vaccine. 2008;26:4218-23. 